\title{
Levetiracetam and Rufinamide are effective at supressing spike and wave seizure activity in an in vitro model of absence epilepsy.
}

Running Title: Levetiracetam and Rufinamide supress Spike and Wave seizures

Virdziniha Todorova ${ }^{1}$, Georgina Ford ${ }^{1}$, Roger.D. Traub ${ }^{2}$, Miles. A. Whittington ${ }^{1}$ and Stephen.

P. Hall ${ }^{1}$,

1. Hull York Medical School, University of York, Heslington, YO10 5DD, UK

2. Dept. of Physical Sciences, IBM T.J. Watson Research Center, Yorktown Heights, NY 10598, USA.

Author for correspondence:

\author{
Stephen Hall \\ HYMS, Biology F1, \\ University of York \\ Heslington, York, YO10 5DD \\ stephen.hall@york.ac.uk
}

Pages =16, Figures = 5. \#words: Abstract: 198, Main text: 3342 .

The authors declare no competing financial interests.

Acknowledgements: We thank The Wellcome Trust for funding this work in the UK. RDT was funded by IBM \& NIH/NINDS R01NS044133. 


\section{Abstract.}

Spike-and-wave discharges ( $\mathrm{SpW}$ ) are seen in absence-type epilepsies. They are heterogeneous in terms of their clinical burden and their electrographic signature, which is used to classify different types of absence seizures; typical absence, in which SpW frequency is $3-4 \mathrm{~Hz}$ and atypical absence, which shows a slower $1-2 \mathrm{~Hz}$ frequency. Treatment of $\mathrm{SpW}$ varies dependent upon the syndrome, but both Valproic Acid (VPA) and Ethosuximide (ESM) are shown to be effective in controlling typical absence seizures. Other anti-epileptic's (AED's), Levetiracetam (LEV) and Rufinamide (RUF), have shown promise in treating absence epilepsies and their associated syndromes. Here we examine the efficacy of these AED's on an in vitro model of SpW.

Both LEV and RUF show an effective reduction in both the number of SpW events and the spike component amplitude; VPA shows no effect, whilst ESM enhances the spike amplitude. Phenytoin exacerbates the SpW activity, increasing both the number of SpW, amplitude of the SpW and the number of spikes within each event. These data suggest that both LEV and RUF could be effective in the treatment of absence-type epilepsies. They also suggest this model could be an effective tool to test other AED's aimed at treating atypical absence syndromes.

Key Words: Absence epilepsy, Anti-epileptic Drugs, Spike-and-Wave, Levetiracetam, Rufinamide 


\section{Introduction}

Spike and wave discharges ( $\mathrm{SpW}$ ) are associated with a wide range of epilepsy pathologies, in particular absence type epilepsies. The concurrent involvement of the thalamus and neocortex is long established (Steriade et al., 1985; 1993; Crunelli \& Leresche, 2002) and several models detailing the dysfunction of this interplay have been shown in which aberrant outputs from thalamic nuclei are emphasised (Destexhe et al., 1993; Destexhe, 1998). However, other studies have described a neocortical focus, showing that abnormal activity in the neocortex comes prior to that in the thalamus (Seneviratne et al., 2014). Our recent work (Hall et al, 2015) developed a model of SpW discharges in an isolated neocortical preparation, highlighting that neocortical networks alone are capable of generating SpW activity.

SpW events are known to be heterogeneous, which means that the SpW frequency, rhythmicity, amplitude distribution and clinical accompaniment can vary dramatically among different seizure pathologies (Blumenfeld, 2005). As such, absence epilepsies which present with SpW are split into two categories; typical absence and atypical absence. Typical SpW is usually described as being a 3$4 \mathrm{~Hz}$ generalized SpW which lasts 10 s of seconds (Gibbs et al., 1935) and are thought to arise from 'normal' sleep spindles (Kostoupolous, 2000). Atypical SpW have been shown at a slower frequency of around $1-2 \mathrm{~Hz}$ and can be seen in secondary generalized epilepsies, such as Lennox-Gastaut syndrome (Niedermeyer, 1968). Whilst clinically there is a challenge to this delineation (Holmes et al., 1987), the roles of both thalamic and neocortical activity in typical and atypical absences is to be debated (Blumenfeld, 2005). Removal of the thalamus, cortex or thalamocortical tract in feline models of $3-4 \mathrm{~Hz}$ generalized seizures leads to the ablation of SpW activity (Avoli \& Gloor, 1981; $1982 ; 1982)$. In contrast, it has been shown that the injection of bicuculline to athalamic rats induces $2-4 \mathrm{~Hz} \mathrm{SpW}$ activity, whilst the injection of bicuculline direct to thalamus does not induce SpW seizures (Steriade \& Contreras, 1998).

The treatment of SpW depends upon the underlying disease pathology but for typical $3-4 \mathrm{~Hz}$ absence SpW, both Valproic acid (VPA) and Ethosuximide (ESM) are effective (Sato et al., 1982), as is Lamotrigine (Schlumberger et al., 1994). Both VPA and ESM have been shown to be more effective than Lamotrigine in controlling $3-4 \mathrm{~Hz}$ typical absence seizures without intolerable side effects (Panayiotopoulos, 2001; Glauser et al., 2010) and as such are currently first treatment options for absence seizures in the NICE guidelines, with Lamotrigine to be considered when both VPA and/or ESM are ineffective or intolerable (NICE Clinical Guidelines, 2012). However, the treatment of slower, $1-2 \mathrm{~Hz}$ atypical absence SpW in diseases such a Lennox-Gastaut is less well described, with NICE guidelines suggesting the use of VPA, for which there is little evidence for its efficacy (Ferrie \& 
Patel, 2009). Novel anti-epileptic drugs (AED's) such as Clobazam (Ng et al, 2011), Rufinamide (Kluger \& Bauer, 2007; Resnik et al., 2011) and Levetiracetam (Verrotti et al., 2008; Fattore et al., 2011) have all shown efficacy in the treatment of childhood and juvenile absence epilepsy syndromes and as such Levetiracetam (LEV) and Rufinamide (RUF) are now listed in the NICE guidelines as therapies to be recommended through referral for absence seizures and for Lennox-Gastaut treatment (NICE Clinical Guidelines, 2012).

Considering the involvement of both the thalamus and the neocortex in clinical SpW, we wanted to examine the pharmacological profile of our neocortical isolated SpW model (Hall et al., 2015). By examining the efficacy of both established typical absence AED's (VPA and ESM) and novel absence AED's (LEV and RUF), combined with examining the effect of Phenytoin (PHE), an AED specifically described as do not prescribe in absence epilepsies (NICE Clinical Guidelines, 2012), we aimed to establish our in vitro SpW model as a specific absence type.

\section{Materials and Methods}

In Vitro electrophysiology: All surgical procedures were performed in accordance with the regulations of the United Kingdom Animals (Scientific Procedures) Act, 1986. Secondary somatosensory/parietal area coronal slices $(450 \mu \mathrm{m}$ thick) were prepared from adult male Wistar rats ( 200g) purchased from Charles River UK. All experiments were carried out between 10am and midday and rats were anaesthetised using isoflurane before terminal anaesthesia was conducted using a cocktail of xylazine and ketamine. A cardiac perfusion was performed before removal of the brain occurred. Slice were maintained at $34^{\circ} \mathrm{C}$ in a standard interface recording chamber containing oxygenated ACSF consisting of (in $\mathrm{mM}$ ): $126 \mathrm{NaCl}, 3 \mathrm{KCl}, 1.25 \mathrm{NaH}_{2} \mathrm{PO}_{4}, 2 \mathrm{MgSO}_{4}, 1.2 \mathrm{CaCl}_{2}, 24$ $\mathrm{NaHCO}_{3}$ and 10 glucose. Persistent, spontaneous delta oscillations were generated as described previously (Carracedo et al., 2013). Once the oscillations were deemed stable (3 recordings over 30 minutes in which the amplitude and frequency did not differ by more than $25 \%$ ) further compounds could be bath applied.

Extracellular field recordings were obtained using micropipettes (2-5 M $\Omega$ ) filled with ACSF and were bandpass filtered at $0.1 \mathrm{~Hz}$ to $200 \mathrm{~Hz}$. Power spectra were derived from Fourier transform analysis of 60 s epochs of data and results were presented as mean \pm SEM. Spike detection and measurement was performed using Axograph and on the basis of transient deflections from the mean membrane voltage. 
Statistical analysis: was performed using SigmaPlot 12.3 (Systat Software Inc.). Normality of the data was tested using the Shapiro-Wilk normality test. For parametric data t-tests were used to measure any differences between the means of data before and after a manipulation. If the difference for more than one group of data was to be calculated a repeated measures Analysis of Variance (ANOVA) Test was used. For non-parametric data, a Mann-Whitney Rank Sum test was used. If the difference for more than one group of data was to be calculated a repeated measures Analysis of Variance (ANOVA) on Ranks was used.

\section{Materials}

Perfusion of the following compounds was performed in certain experiments; (+)Tubocurarine chloride $\left(10 \mu \mathrm{M}\right.$, antagonist to nicotinic acetylcholine and 5- $\mathrm{HT}_{3}$ receptors), BMS193885 (10 $\mu \mathrm{M}$, antagonist to NPY receptors), [D-p-Cl-Phe6, Leu17]-VIP (1 $\mu \mathrm{M}$, Agonist to VIP receptors),

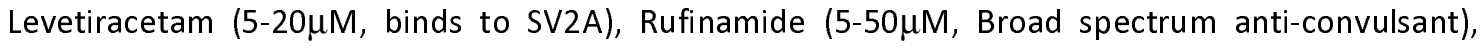
Phenytoin $(5-20 \mu \mathrm{M}$, blocks voltage gated sodium channels), Valproic Acid, sodium salt (5-20 $\mu \mathrm{M}$, Blocks voltage gated sodium channels), Ethosuximide (5-20 $\mu \mathrm{M}$, blocks T-type calcium channels). Drugs were obtained from Tocris Bioscience or Sigma Aldrich.

\section{Results}

The effect of AED's upon delta rhythms in the secondary somatosensory cortex

The incidence of SpW has been shown to markedly increase with sleep (Angeleri et al., 1968). Previous work has highlighted that generating SpW in vitro is exquisitely dependent upon a background of delta frequency oscillations (Hall et al., 2015). Therefore, in order to examine the efficacy of AED's on our model, it was first imperative to examine their effect upon delta oscillations.

The bath application of $5 \mu \mathrm{M}, 10 \mu \mathrm{M}$ or $20 \mu \mathrm{M}$ of ESM (Fig. 1A) showed no significant effects $(\mathrm{P}>0.05$. $\mathrm{n}=5$ slices, 3 animals for all concentrations) upon the amplitude $(5 \mu \mathrm{M} ; 142.45 \mu \mathrm{V} \pm 82.36$ vs. $189.3 \mu \mathrm{V}$ $\pm 106.65,10 \mu \mathrm{M} ; 195.96 \mu \mathrm{V} \pm 76.74$ vs. $172.48 \mu \mathrm{V} \pm 69.58,20 \mu \mathrm{M} ; 402.28 \mu \mathrm{V} \pm 131.98$ vs. $499.48 \mu \mathrm{V} \pm$ 163.09) or the frequency $(5 \mu \mathrm{M} ; 1.46 \mathrm{~Hz} \pm 0.1$ vs. $1.46 \mathrm{~Hz} \pm 0.09,10 \mu \mathrm{M} ; 1.32 \mathrm{~Hz} \pm 0.11$ vs. $1.25 \mathrm{~Hz} \pm$ $0.1,20 \mu \mathrm{M} ; 1.27 \mathrm{~Hz} \pm 0.3 v s .1 .27 \mathrm{~Hz} \pm 0.1$ ) of the delta oscillations. The bath application of $5 \mu \mathrm{M}$, $10 \mu \mathrm{M}$ or $20 \mu \mathrm{M}$ of VPA (Fig. 1B) again showed no significant effects ( $P>0.05 . n=5$ slices, 3 animals for $5 \mu \mathrm{M}$; 6 slices, 3 animals for $10 \mu \mathrm{M}$ and $20 \mu \mathrm{M})$ upon the amplitude $(5 \mu \mathrm{M} ; 247.98 \mu \mathrm{V} \pm 137.28$ vs. $206.9 \mu \mathrm{V} \pm 129.87,10 \mu \mathrm{M} ; 154.9 \mu \mathrm{V} \pm 77.14$ vs. $164.12 \mu \mathrm{V} \pm 66.74,20 \mu \mathrm{M} ; 100.87 \mu \mathrm{V} \pm 28.65$ vs. 
$138.97 \mu \mathrm{V} \pm 40.16)$ or the frequency $(5 \mu \mathrm{M} ; 1.41 \mathrm{~Hz} \pm 0.13$ vs. $1.41 \mathrm{~Hz} \pm 0.1,10 \mu \mathrm{M} ; 1.63 \mathrm{~Hz} \pm 0.15$ vs. $1.63 \mathrm{~Hz} \pm 0.07,20 \mu \mathrm{M} ; 1.54 \mathrm{~Hz} \pm 0.12$ vs. $1.39 \mathrm{~Hz} \pm 0.1$ ) of the delta oscillations.

The bath application of $5 \mu \mathrm{M}, 10 \mu \mathrm{M}$ or $20 \mu \mathrm{M}$ of LEV (Fig. 1C) showed no significant effects ( $>0.05$. $\mathrm{n}=6$ slices, 4 animals for $5 \mu \mathrm{M} ; 7$ slices, 5 animals for $10 \mu \mathrm{M}$ and $20 \mu \mathrm{M})$ upon the amplitude $(5 \mu \mathrm{M}$; $254.0 \mu \mathrm{V} \pm 83.28$ vs. $228.62 \mu \mathrm{V} \pm 105.58,10 \mu \mathrm{M} ; 252.3 \mu \mathrm{V} \pm 143.26$ vs. $226.99 \mu \mathrm{V} \pm 118.04,20 \mu \mathrm{M}$; $303.27 \mu \mathrm{V} \pm 76.15$ vs. $322.18 \mu \mathrm{V} \pm 66.59)$ or the frequency $(5 \mu \mathrm{M} ; 1.35 \mathrm{~Hz} \pm 0.24$ vs. $1.34 \mathrm{~Hz} \pm 0.17$, $10 \mu \mathrm{M} ; 1.82 \mathrm{~Hz} \pm 0.21$ vs. $1.74 \mathrm{~Hz} \pm 0.2,20 \mu \mathrm{M} ; 1.57 \mathrm{~Hz} \pm 0.09$ vs. $1.46 \mathrm{~Hz} \pm 0.07$,) of the delta oscillations. The bath application of $5 \mu \mathrm{M}, 10 \mu \mathrm{M}$ or $20 \mu \mathrm{M}$ of RUF (Fig. 1D) again showed no significant effects ( $P>0.05$. $n=6$ slices, 4 animals for all concentrations) upon the amplitude $(5 \mu \mathrm{M}$; $427.61 \mu \mathrm{V} \pm 110.17$ vs. $482.51 \mu \mathrm{V} \pm 176.58,10 \mu \mathrm{M} ; 133.69 \mu \mathrm{V} \pm 37.27$ vs. $109.59 \mu \mathrm{V} \pm 29.35,20 \mu \mathrm{M}$; $192.67 \mu \mathrm{V} \pm 49.56$ vs. $245.04 \mu \mathrm{V} \pm 80.29)$ or the frequency $(5 \mu \mathrm{M} ; 1.26 \mathrm{~Hz} \pm 0.15$ vs. $1.11 \mathrm{~Hz} \pm 0.13$, $10 \mu \mathrm{M} ; 1.85 \mathrm{~Hz} \pm 0.17$ vs. $1.73 \mathrm{~Hz} \pm 0.16,20 \mu \mathrm{M} ; 1.42 \mathrm{~Hz} \pm 0.09$ vs. $1.2 \mathrm{~Hz} \pm 0.17$ ) of the delta oscillations.

Finally, the bath application of $5 \mu \mathrm{M}, 10 \mu \mathrm{M}$ or $20 \mu \mathrm{M}$ of phenytoin (Fig. 1E) showed no significant effects ( $P>0.05 . n=6$ slices, 3 animals for all concentrations) upon the amplitude ( $5 \mu \mathrm{M} ; 199.92 \mu \mathrm{V} \pm$ 72.3 vs. $181.4 \mu \mathrm{V} \pm 45.1,10 \mu \mathrm{M} ; 120.65 \mu \mathrm{V} \pm 27.66$ vs. $129.87 \mu \mathrm{V} \pm 51.52,20 \mu \mathrm{M} ; 229.32 \mu \mathrm{V} \pm 72.16$ vs. $186.28 \mu \mathrm{V} \pm 48.61)$ or the frequency $(5 \mu \mathrm{M} ; 2.08 \mathrm{~Hz} \pm 0.23$ vs. $1.89 \mathrm{~Hz} \pm 0.23,10 \mu \mathrm{M} ; 1.78 \mathrm{~Hz} \pm 0.17 v s$. $1.51 \mathrm{~Hz} \pm 0.21,20 \mu \mathrm{M} ; 1.69 \mathrm{~Hz} \pm 0.07$ vs. $1.52 \mathrm{~Hz} \pm 0.15$ ) of the delta oscillations.

The effect of established AED's on SpW activity in the secondary somatosensory cortex.

The NICE guidelines suggest the use of ESM, VPA and lamotrigine, either in mono-therapy or in combination therapy to supress absence type seizure. Studies have highlighted the greater efficacy and reduced side effects offered by ESM and VPA when compared to lamotrigine (Glauser et al., 2010; 2013). Given this, we decided to examine the effect of ESM and VPA upon SpW activity, generated as per Hall et al., 2015. On a background of persistent delta oscillations we generated $\mathrm{SpW}$ activity in both control conditions, with the bath application of d-TC alone and by bath applying $\mathrm{d}-\mathrm{TC}$ in the presence of varying concentrations of ESM and VPA. We then examined the number of $\mathrm{SpW}$ events per minute and the amplitude of the spike component of the SpW event.

Bath application of $5 \mu \mathrm{M}, 10 \mu \mathrm{M}$ or $20 \mu \mathrm{M}$ ESM prior to the generation of SpW activity failed to supress seizure activity (Fig. $2 \mathrm{~A}, \mathrm{~B}, \mathrm{Ei}$ ). At $5 \mu \mathrm{M}$, the number of $\mathrm{SpW}$ events per minute did not significantly differ, averaging $10.29 \mathrm{~min}^{-1} \pm 0.67$ with $\mathrm{d}-\mathrm{TC}$ alone and $11.00 \mathrm{~min}^{-1} \pm 1$ with ESM $(P>0.05$, 
$\mathrm{n}=5$ slices $/ 3$ animals). Again, no difference was observed at either $10 \mu \mathrm{M}$ ESM $\left(11.40 \mathrm{~min}^{-1} \pm 0.92\right.$; $P>0.05, n=5$ slices $/ 3$ animals) or $20 \mu M$ ESM $\left(11.60 \mathrm{~min}^{-1} \pm 0.36 ; P>0.05, n=5\right.$ slices $/ 3$ animals). The amplitude of the spike component did show a significant increase at $5 \mu \mathrm{M}$ ESM, averaging at $423.29 \mu \mathrm{V} \pm 46.96$ with $\mathrm{d}-\mathrm{TC}$ alone and $769.78 \mu \mathrm{V} \pm 92.4$ with $\mathrm{ESM}(\mathrm{P}=0.016, \mathrm{n}=5$ slices $/ 3$ animals). This increase was sustained but non-significant at $10 \mu \mathrm{M}(608.2 \mu \mathrm{V} \pm 184.29 ; \mathrm{P}>0.05, \mathrm{n}=5$ slices $/ 3$ animals), but was significant once more at $20 \mu \mathrm{M}(740.6 \mu \mathrm{V} \pm 83.13 ; \mathrm{P}=0.022, \mathrm{n}=5$ slices $/ 3$ animals $)$.

Bath application of $5 \mu \mathrm{M}, 10 \mu \mathrm{M}$ or $20 \mu \mathrm{M}$ VPA prior to the generation of SpW activity failed to supress seizure activity (Fig. 2 C, D, Eii). At $5 \mu \mathrm{M}$, the number of SpW events per minute showed no significant change, averaging $10.29 \mathrm{~min}^{-1} \pm 0.67$ with $\mathrm{d}$-TC alone and $11.00 \mathrm{~min}^{-1} \pm 1.1$ with VPA $\left(P>0.05, n=5\right.$ slices $/ 3$ animals). Again, no difference was observed at either $10 \mu \mathrm{M}$ VPA $\left(9.5 \mathrm{~min}^{-1} \pm\right.$ $0.57 ; P>0.05, n=6$ slices $/ 3$ animals) or $20 \mu M$ VPA $\left(8.83 \mathrm{~min}^{-1} \pm 0.48 ; P>0.05, n=6\right.$ slices $/ 3$ animals). The amplitude of the spike component also showed no significant change at $5 \mu \mathrm{M}$ ESM, averaging at $423.29 \mu \mathrm{V} \pm 46.96$ with $\mathrm{d}-\mathrm{TC}$ alone and $512.23 \mu \mathrm{V} \pm 78.78$ with VPA ( $>0.05, n=5$ slices $/ 3$ animals). Again, no differences were observed at $10 \mu \mathrm{M}(419.08 \mu \mathrm{V} \pm 125.17 ; \mathrm{P}>0.05, \mathrm{n}=6$ slices $/ 3$ animals $)$ or $20 \mu \mathrm{M}(361.17 \mu \mathrm{V} \pm 29.6 ; \mathrm{P}>0.05, \mathrm{n}=6$ slices $/ 3$ animals $)$.

The effect of novel AED's on SpW activity in the secondary somatosensory cortex.

LEV has been shown to provide seizure suppression and an increase in cognitive function in patients with Lennox-Gastaut syndrome (De Los Reyes et al., 2004; Diaz-Negrillo et al., 2011) as has RUF (Resnik et al., 2011). Given this, we decided to challenge our SpW activity, generated as per Hall et al., 2015, with LEV and RUF. On a background of persistent delta oscillations we generated SpW activity in both control conditions, with the bath application of $\mathrm{d}$-TC alone, and by bath applying $\mathrm{d}$-TC in the presence of varying concentrations of LEV and RUF. We then examined the number of SpW events per minute and the amplitude of the spike component of the SpW event.

Bath application of all concentrations of LEV prior to the generation of SpW activity successfully supressed seizure activity (Fig. 3 A, B, Ei), averaging $10.29 \mathrm{~min}^{-1} \pm 0.67$ with $\mathrm{d}-\mathrm{TC}$ alone and $6.00 \mathrm{~min}^{-1}$ \pm 0.97 with LEV ( $P<0.05, n=6$ slices/4 animals). At $10 \mu \mathrm{M}$, LEV further reduced SpW activity $\left(1.29 \mathrm{~min}^{-}\right.$ ${ }^{1} \pm 0.61 ; \mathrm{P}<0.05, \mathrm{n}=7$ slices $/ 5$ animals) and at $20 \mu \mathrm{M}$, LEV had completely supressed SpW events $\left(0 \mathrm{~min}^{-1} \pm 0 ; \mathrm{P}<0.05, \mathrm{n}=5\right.$ slices $/ 4$ animals). The amplitude of the spike component did not show a significant change at $5 \mu \mathrm{M} \mathrm{LEV}$, averaging at $423.29 \mu \mathrm{V} \pm 46.96$ with $\mathrm{d}-\mathrm{TC}$ alone and $761.11 \mu \mathrm{V} \pm$ 301.62 with LEV ( $P>0.05, n=6$ slices/4 animals). However, at 10 $\mu \mathrm{M}$ there was a significant reduction in the amplitude of the spike $(183.68 \mu \mathrm{V} \pm 118.84 ; \mathrm{P}<0.05, \mathrm{n}=7$ slices $/ 5$ animals $)$ and given the $\mathrm{SpW}$ 
suppression, the lack of SpW meant a significant reduction at $20 \mu \mathrm{M}(0 \mu \mathrm{V} \pm 0 ; \mathrm{P}<0.001, \mathrm{n}=7$ slices $/ 4$ animals).

Bath application of high concentrations of RUF prior to the generation of SpW activity also supressed seizure activity (Fig. 3 C, D, Eii). At $5 \mu \mathrm{M}$, the number of SpW events per minute showed no significant change, averaging $10.29 \mathrm{~min}^{-1} \pm 0.67$ with $d-T C$ alone and $12.33 \mathrm{~min}^{-1} \pm 1.61$ with RUF $(P>0.05, n=6$ slices/4 animals). Again, no difference was observed at $10 \mu \mathrm{M}$ RUF $\left(7.17 \mathrm{~min}^{-1} \pm 1.61 ; \mathrm{P}>0.05, \mathrm{n}=6\right.$

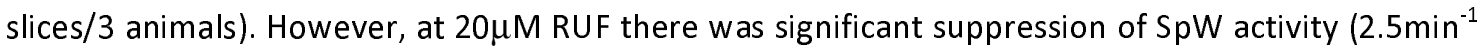
$\pm 1.15 ; \mathrm{P}<0.05, \mathrm{n}=6$ slices $/ 5$ animals). The amplitude of the spike component showed no significant change at $5 \mu \mathrm{M}$ RUF, averaging at $423.29 \mu \mathrm{V} \pm 46.96$ with $\mathrm{d}-\mathrm{TC}$ alone and $496.89 \mu \mathrm{V} \pm 111.49$ with RUF $(P>0.05, n=6$ slices $/ 4$ animals). Again, no differences were observed at $10 \mu \mathrm{M}(456.87 \mu \mathrm{V} \pm 119.38$; $\mathrm{P}>0.05, \mathrm{n}=6$ slices $/ 3$ animals), but at $20 \mu \mathrm{M}$ RUF there was a significant reduction in the amplitude of the spike $(111.78 \mu \mathrm{V} \pm 45.92 ; \mathrm{P}<0.05, \mathrm{n}=6$ slices $/ 5$ animals $)$.

The effect of phenytoin on SpW activity in the secondary somatosensory cortex.

PHE is currently listed as do not prescribe for absence type seizures (NICE guidelines 2012). We wanted to examine its effect upon our in vitro model of SpW events to determine if it had an exacerbating effect upon the SpW activity. On a background of persistent delta oscillations we generated SpW activity in both control conditions, with the bath application of d-TC alone and by bath applying $d-T C$ in the presence of varying concentrations of PHE.

Bath application of high concentrations of PHE prior to the generation of SpW activity caused an increase to both SpW number and spike amplitude (Fig. 4 A, B, D). At $5 \mu \mathrm{M}$, the number of SpW events per minute showed no significant change, averaging $10.29 \mathrm{~min}^{-1} \pm 0.67$ with $\mathrm{d}$-TC alone and $11.2 \mathrm{~min}^{-1} \pm 1.4$ with PHE ( $>0.05, \mathrm{n}=5$ slices/3 animals). Again, no difference was observed at $10 \mu \mathrm{M}$ PHE (9.33 $\mathrm{min}^{-1} \pm 1.31 ; \mathrm{P}>0.05, \mathrm{n}=6$ slices $/ 3$ animals). However, at $20 \mu \mathrm{M}$ PHE there was a significant increase in SpW activity $\left(15.5 \mathrm{~min}^{-1} \pm 2 ; \mathrm{P}<0.05, \mathrm{n}=6\right.$ slices $/ 3$ animals). The amplitude of the spike component showed significant change at $5 \mu \mathrm{M} \mathrm{PHE}$, averaging at $423.29 \mu \mathrm{V} \pm 46.96$ with $\mathrm{d}-\mathrm{TC}$ alone and $797 \mu \mathrm{V} \pm 62.6$ with PHE ( $\mathrm{P}<0.05, \mathrm{n}=5$ slices $/ 3$ animals). This increase in amplitude was enhanced further at $10 \mu \mathrm{M}$ PHE $(1819.33 \mu \mathrm{V} \pm 520.74 ; \mathrm{P}<0.001, \mathrm{n}=6$ slices $/ 3$ animals $)$, and at $20 \mu \mathrm{M}(1543.33 \mu \mathrm{V}$ $\pm 229.18 ; \mathrm{P}<0.001, \mathrm{n}=6$ slices $/ 3$ animals).

In testing all other AED's only a single spike was observed within each SpW event. However, with the bath application of PHE, instances of multiple spikes were observed within each SpW (Fig. 4 C, E). At $5 \mu \mathrm{M}$ PHE, the number of spikes per SpW event increased from $1 \pm 0$ with $\mathrm{d}-\mathrm{TC}$ alone, to $1.27 \pm 0.11$ with PHE. This increase was maintained at $10 \mu \mathrm{M}$ PHE $(1.28 \pm 0.09)$ and at $20 \mu \mathrm{M}(1.45 \pm 0.13)$. 
However, none of the increases in spike number were significant $(P>0.05 . n=5$ slices/3animals for $5 \mu \mathrm{M}$ and $10 \mu \mathrm{M}, \mathrm{n}=6$ slices/3animals for $20 \mu \mathrm{M})$.

The effect of Levetiracetam on SpW activity induced by peptidergic disinhibition, in the secondary somatosensory cortex.

In Hall et al, 2015, we explored the mechanisms behind the development of SpW. It was observed that SpW was caused by the disruption in the balance between two peptides, neuropeptide Y (NPY) and vasointestinal peptide (VIP). Using a combination of an NPY antagonist (10 $\mu \mathrm{M}$ BMS193885) and $1 \mu \mathrm{M}$ VIP on a background of persistent delta oscillations, SpW was observed. The most overt effect of the AED's on d-TC induced SpW activity was with LEV. We wanted to test the effect of LEV on the peptidergic model of SpW activity, to examine if its effect was maintained in this more physiological model.

Bath application of $20 \mu \mathrm{M}$ LEV prior to the generation of $\mathrm{SpW}$ activity caused a significant reduction in both SpW number (Fig. 5 A, B, D). The number of SpW events reduced from $16.29 \mathrm{~min}^{-1} \pm 1.19$ with BMS 193855 and VIP and $1 \mathrm{~min}^{-1} \pm 0.43$ with $20 \mu \mathrm{M}$ LEV $(\mathrm{P}<0.05, \mathrm{n}=8$ slices/4 animals BMS 193855/VIP, $n=7$ slices/4 animals 20 MM LEV). Whilst the number of SpW per minute was reduced with the addition of LEV, the SpW amplitude was not significantly reduced. The amplitude of the spike component also showed a non-significant decrease from $106.8 \mu \mathrm{V} \pm 20.92$ with BMS 193885 and VIP and $70.75 \mu \mathrm{V} \pm 20.13$ with $20 \mu \mathrm{M} \operatorname{LEV}(\mathrm{P}>0.05, \mathrm{n}=8$ slices/4 animals BMS 193855/NIP, $\mathrm{n}=7$ slices/4 animals $20 \mu \mathrm{M}$ LEV).

\section{Discussion}

The interplay between the thalamus and cortex in the development of SpW activity is long established. However, the role of the thalamus and/or cortex in different SpW types is complex. The removal of the thalamus, cortex or thalamocortical tract in feline models of $3-4 \mathrm{~Hz}$ generalized seizures leads to the ablation of SpW activity (Avoli \& Gloor, 1981; 1982; 1982), whilst in genetic absence epilepsy rats of Strasbourg (GAERS), faster SpW (c7-11Hz) cannot be sustained with the removal of either thalamus or neocortex (Danober et al., 1998). Furthermore, mean field modelling of the corticothalamic system shows that increasing the feed-forward inhibition of cortex to thalamic relay neurons can supress $3-4 \mathrm{~Hz} \mathrm{SpW}$ activity (Chen et al., 2017). These studies seem to suggest a role for both thalamus and neocortex in typical absence seizures. Conversely, SpW activity when the thalamus is ablated and when cortex is isolated has also been described (Steriade and 
Contreras, 1998; Timofeev \& Steriade, 2004). However, some of the SpW activity within these models appears to be at the slower $1-2 \mathrm{~Hz}$ frequency more associated with atypical absence. Given this evidence, it has been postulated that the involvement of the thalamus in the generation of $\mathrm{SpW}$ may depend upon the model (Blumenfeld, 2005). The model studied here is an isolated neocortical model; the thalamus plays no role in the generation of SpW.

SpW have been shown to increase with sleep (Angeleri et al., 1968) and the dependence upon delta frequency activity to generate SpW in this model was highlighted in Hall et al., 2015. The data within this study show that no AED's tested have any significant effect upon the delta rhythm in vitro, allowing us to be sure we are testing their efficacy upon the SpW events. Both of the AED's used to treat typical absence seizures, VPA and ESM, had no positive effect upon SpW activity. ESM, whilst not altering the number of SpW events observed, did increase the amplitude of the spike component of the events. The spike component of the event in this model has been shown to be generated from the superficial layers of the cortex (Hall et al., 2015). As such an increase in the amplitude of the spike component suggests a shift from cells firing single spikes, to firing bursts. Thus the increase in the spike amplitude suggests that ESM is exacerbating the SpW, akin to the effect of PHE in this model. PHE increased the SpW number at high doses and increased the amplitude of the spike component throughout the concentration range. More significantly PHE caused an increase in the number of spikes within each event at all doses, highlighting a distinct exacerbation of SpW as previously considered (Genton, 2000). As such it appears correct that PHE is on the do not prescribe list for absence type epilepsies.

LEV showed effective SpW reduction, both in term of SpW number and in terms of the spike component amplitude. Patient studies have shown LEV improves atypical absence seizures observed in disorders such as Lennox-Gastaut (De Los Reyes et al., 2004), Angelman syndrome (Shaaya et al., 2016) and Jeavons syndrome (Striano et al., 2008). It also has been shown to improve the cognitive deficits associated with Lennox-Gastaut syndrome (Diaz-Negrillo et al., 2011). However, it has been described as aggravating typical absence seizures (Auvin et al., 2011). The other AED to show effective reductions in SpW within this study was RUF. Again, RUF is effective in the treatment atypical absence seizures (Albini et al., 2016) particularly in diseases such as Lennox-Gastaut (Glauser et al., 2008) and as such it has recently been approved as an adjunctive therapy.

The model used in this study uses isolated neocortex to generate SpW and given the evidence discussed above the model could be described as being atypical in its presentation. A dramatic reduction in SpW observed with the application of LEV and RUF, which have shown efficacy in patients with atypical absence epilepsy but not in patients with typical absence, was observed. 
Couple this with the lack of efficacy of VPA and ESM, which are both prescribed for typical absence epilepsies and the evidence would suggest that the in vitro model used in this study and first described in Hall et al, 2015 is of atypical absence epilepsy. Given the slower frequency of atypical absence epilepsies discussed in the introduction, this would potentially explain the slower frequency SpW activity observed in this model (Hall et al., 2015). The implications of this study are two-fold: 1) It further highlights the excellent efficacy of LEV and RUF in the treatment of atypical absence epilepsies; 2) Given the difficulties in treating atypical absence epilepsies (Sinclair and Unwala, 2007) this model could provide an effective tool to test novel AED's aimed at treating atypical absence epilepsies.

\section{References}

Albini M, Morano A, Fanella M, Lapenta L, Casciato S, Fattouch J, Manfredi M, Giallonardo AT, Di Bonaventura C. Effectiveness of Rufinamide in the Treatment of Idiopathic Generalized Epilepsy With Atypical Evolution: Case Report and Review of the Literature. Clin EEG Neurosci. 2016 Apr;47(2):162-6.

Angeleri F, Berbonzi P, Ferroni, A. A statistical study of the amount of diffuse synchronous spike and wave activity during nocturnal polygraphic recording in epileptics. Electroencephalogr Clin Neurophysiol. 1968, 25: 85-6.

Auvin S, Chhun S, Berquin P, Ponchel E, Delanoë C, Chiron C. Aggravation of absence seizure related to levetiracetam. Eur J Paediatr Neurol. 2011, Nov; 15(6): 508-11.

Avoli M, Gloor P. The effects of transient functional depression of the thalamus on spindles and on bilateral synchronous epileptic discharges of feline generalized penicillin epilepsy. Epilepsia. 1981, 22(4):443-52.

Avoli M, Gloor P. Role of the thalamus in generalized penicillin epilepsy: observations on decorticated cats. Exper Neurol. 1982, 77(2):386-402.

Avoli M, Gloor P. Interaction of cortex and thalamus in spike and wave discharges of feline generalized penicillin epilepsy. Exper Neurol. 1982, 76(1):196-217.

Blumenfeld H. Cellular and network mechanisms of spike-wave seizures. Epilepsia. 2005, 46:21-33.

Brigo F, Igwe SC. Ethosuximide, sodium valproate or lamotrigine for absence seizures in children and adolescents. Cochrane Database Syst Rev. 2017, Feb 14;2:CD003032. 
Carracedo LM, Kjeldsen $\mathrm{H}$, Cunnington L, et al. A neocortical delta rhythm facilitates reciprocal interlaminar interactions via nested theta rhythms. J Neurosci. 2013, 33:10750-61.

Crunelli V, Leresch, N. Childhood absence epilepsy: genes, channels, neurons and networks. Nat Rev Neurosci. 2002, 3:371-82.

Danober L, Depaulis A, Marescaux C, Vergnes M. Effects of cholinergic drugs on genetic absence seizures in rats. Eur J Pharmacol. 1993, 234: 263-8.

De Los Reyes EC, Sharp GB, Williams JP, Hale SE. Levetiracetam in the treatment of Lennox-Gastaut syndrome. Pediatr Neurol. 2004, Apr;30(4):254-6.

Destexhe A, McCormick DA, Sejnowski TJ Thalamic and thalamocortical mechanisms underlying $3 \mathrm{~Hz}$ spike-and-wave discharges. Prog Brain Res. 1999, 121: 289-307.

Destexhe A. Spike-and-wave oscillations based on the properties of GABAB receptors. J Neurosci. 1998, 18: 9099-111.

Díaz Negrillo A, Martín Del Valle F, González Salaices M, Prieto Jurczynska C, Carneado Ruiz J. Levetiracetam efficacy in patients with Lennox-Gastaut syndrome. Presentation of a case. Neurologia. 2011, Jun;26(5):285-90.

Fattore, C., Boniver, C., Capovilla, G, Cerminara C, Citterio A, Coppola G, Costa P, Darra F, Vecchi M, Perucca E. A multicenter, randomized, placebo-controlled trial of levetiracetam in children and adolescents with newly diagnosed absence epilepsy. Epilepsia. 2011, 52: 802-809

Ferrie CD, Patel A. Treatment of Lennox-Gastaut Syndrome (LGS). Eur J Paediatr Neurol. 2009, Nov;13(6):493-504.

Genton, P. When antiepileptic drugs aggravate epilepsy. Brain Dev. 2000, Mar; 22 (2): 75-80.

Gibbs FA, Davis H, Lennox WG. The electroencephalogram in epilepsy and in conditions of impaired consciousness. Arch Neurol Psychiatr. 1935, 34:1133-1148.

Glauser T, Kluger G, Sachdeo R, Krauss G, Perdomo C, Arroyo S.Rufinamide for generalized seizures associated with Lennox-Gastaut syndrome. Neurology. 2008, May 20;70(21):1950-8.

Glauser TA, Cnaan A, Shinnar S, Hirtz DG, Dlugos D, Masur D, Clark PO, Capparelli EV, Adamson PC; Childhood Absence Epilepsy Study Group. Ethosuximide, valproic acid, and lamotrigine in childhood absence epilepsy. N Engl J Med. 2010, Mar 4;362(9):790-9. 
Glauser TA, Cnaan A, Shinnar S, Hirtz DG, Dlugos D, Masur D, Clark PO, Adamson PC; Childhood Absence Epilepsy Study Team. Childhood Absence Epilepsy Study Team. Ethosuximide, valproic acid, and lamotrigine in childhood absence epilepsy: initial monotherapy outcomes at 12 months. Epilepsia. 2013, 54(1):141-55.

Hall S, Hunt M, Simon A et al. Unbalanced Peptidergic Inhibition in Superficial Neocortex Underlies Spike and Wave Seizure Activity. J Neurosci. 2015, 35:9302-14.

Holmes $G L$, McKeever $M$, Adamson $M$. Absence seizures in children: clinical and electroencephalographic features. Ann Neurol. 1987, 21:268-73.

Kluger G, Bauer B. Role of rufinamide in the management of Lennox-Gastaut syndrome (childhood epileptic encephalopathy). Neuropsychiatr Dis Treat. 2007, Feb;3(1):3-11.

Kostopoulos G. Spike and wave discharges of absence seizures as a transformation of sleep spindles: the continuing development of a hypothesis. Clin Neurophysiol. 2000, 111 (suppl 2) :S27-38.

Ng YT, Conry JA, Drummond R, Stolle J, Weinberg MA; OV-1012 Study Investigators. Randomized, phase III study results of clobazam in Lennox-Gastaut syndrome. Neurology. 2011, Oct $11 ; 77(15): 1473-81$

Niedermeyer E. The Lennox-Gastaut syndrome: a severe type of childhood epilepsy. Electroencephalogr Clin Neurophysiol. 1968, 24:283.

Panayiotopoulos C P. Treatment of typical absence seizures and related epileptic syndromes. Paediatric Drugs. 2001, 3(5):379-403.

Resnick T, Arzimanoglou A, Brown LW, Flamini R, Kerr M, Kluger G, Kothare S, Philip S, Harrison M, Narurkar M. Rufinamide from clinical trials to clinical practice in the United States and Europe. Epileptic Disord. 2011, May;13 Suppl 1:S27-43.

Sato S, White BG, Penry JK, Dreifuss FE, Sackellares JC, Kupferberg HJ. Valproic acid versus ethosuximide in the treatment of absence seizures. Neurology, 1982;32(2):157-63.

Seneviratne U, Cook M, D'Souza W. Focal abnormalities in idiopathic generalized epilepsy: A critical review of the literature. Epilepsia. 2014; 55:1157-69.

Shaaya EA, Grocott OR, Laing O, Thibert RL. Seizure treatment in Angelman syndrome: A case series from the Angelman Syndrome Clinic at Massachusetts General Hospital.Epilepsy Behav. 2016 Jul;60:138-41. 
Sinclair $\mathrm{DB}^{1}$, Unwala H.Absence epilepsy in childhood: electroencephalography (EEG) does not predict outcome. J Child Neurol. 2007 Jul;22(7):799-802.

Steriade M, Deschênes M, Domich L, Mulle C. Abolition of spindle oscillations in thalamic neurons disconnected from nucleus reticularis thalami. J Neurophysiol. 1985, 54:1473-1497.

Steriade M, McCormick DA, Sejnowski TJ Thalamocortical oscillations in the sleeping and aroused brain. Science. 1993, 262: 679-685.

Steriade M, Contreras D. Spike-wave complexes and fast components of cortically generated seizures. I. Role of neocortex and thalamus. J Neurophysiol. 1998, 80(3):1439-55.

Striano P, Sofia V, Capovilla G, Rubboli G, Di Bonaventura C, Coppola A, Vitale G, Fontanillas L, Giallonardo AT, Biondi R, Romeo A, Viri M, Zara F, Striano S. A pilot trial of levetiracetam in eyelid myoclonia with absences (Jeavons syndrome). Epilepsia. 2008, Mar;49(3):425-30.

Verrotti A, Cerminara C, Domizio S, Mohn A, Franzoni E, Coppola G, Zamponi N, Parisi P, lannetti P, Curatolo P. Levetiracetam in absence epilepsy. Dev Med Child Neurol. 2008, Nov;50 (11):850-3.

\section{Figure Legends}

Figure 1. VPA, ESM, LEV, RUF and PHE have no effect on delta amplitude or frequency. Ai. Bar chart showing ESM had no effect upon delta amplitude at any of the concentration points. Solid bar = before ESM, transparent bar = after addition of ESM. Aii. Bar chart showing ESM had no effect upon delta frequency at any of the concentration points. Solid bar = before ESM, transparent bar = after addition of ESM. Bi. Bar chart showing VPA had no effect upon delta amplitude at any of the concentration points. Solid bar $=$ before VPA, transparent bar $=$ after addition of VPA. Bii. Bar chart showing VPA had no effect upon delta frequency at any of the concentration points. Solid bar = before VPA, transparent bar = after addition of VPA. Ci. Bar chart showing LEV had no effect upon delta amplitude at any of the concentration points. Solid bar = before LEV, transparent bar = after addition of LEV. Cii. Bar chart showing LEV had no effect upon delta frequency at any of the concentration points. Solid bar = before LEV, transparent bar = after addition of LEV. Di. Bar chart showing RUF had no effect upon delta amplitude at any of the concentration points. Solid bar = before RUF, transparent bar = after addition of LEV. Dii. Bar chart showing RUF had no effect upon delta frequency at any of the concentration points. Solid bar = before RUF, transparent bar = after addition of RUF. Ei. Bar chart showing PHE had no effect upon delta amplitude at any of the 
concentration points. Solid colour bar = before PHE, transparent bar = after addition of PHE. Eii. Bar chart showing PHE had no effect upon delta frequency at any of the concentration points. Solid bar = before PHE, transparent bar = after addition of PHE.

Figure 2. ESM and VPA show no suppression of d-TC induced SpW activity. A. Line graph showing ESM has no effect upon SpW number. B. Line graph showing ESM has an exacerbating effect upon the amplitude of the spike component of SpW at 5 and $20 \mu \mathrm{M}$. C. Line graph showing VPA has no effect upon SpW number. D. Line graph showing VPA has no effect upon the amplitude of the spike component of SpW. E. Example trace showing SpW activity recorded from S2 in the presence of $20 \mu \mathrm{M}$ ESM. F. Example trace showing SpW activity recorded from S2 in the presence of $20 \mu \mathrm{M}$ VPA. Scale bar $=200 \mu \mathrm{V}, 10$ seconds. ${ }^{*}$ indicates $\mathrm{P}<0.05$.

Figure 3. LEV and RUF show effective suppression of d-TC induced SpW activity. A. Line graph showing the reduction in SpW number, which correlates with increasing concentrations of LEV. B. Line graph showing LEV reduces the amplitude of the spike component of SpW at 5 and $20 \mu \mathrm{M}$. C.

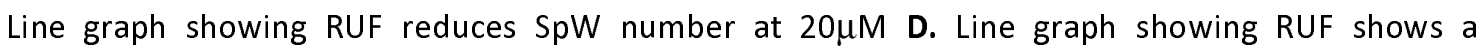
significant reduction in SpW amplitude at 20 $\mathrm{MM}$. E. Example trace showing SpW activity recorded from S2 in the presence of $20 \mu \mathrm{M}$ LEV. F. Example trace showing SpW activity recorded from S2 in the presence of $20 \mu \mathrm{M}$ RUF. Scale bar $=100 \mu \mathrm{V}, 10$ seconds. ${ }^{*}$ indicates $\mathrm{P}<0.05 .{ }^{* *}$ indicates $\mathrm{P}<0.001$.

Figure 4. PHE exacerbates d-TC induced SpW activity. A. Line graph showing an increase in SpW number at $20 \mu \mathrm{M}$ PHE. B. Line graph showing PHE significantly increases the amplitude of the spike component of SpW at all concentration points. C. Bar chart showing an increase in the number of spikes per event when SpW were induced by d-TC in the presence of PHE. Clear bar show number of spikes per SpW event in d-TC alone, coloured bars in the presence of PHE. D. Example trace showing $\mathrm{SpW}$ activity recorded from S2 in the presence of $20 \mu \mathrm{M}$ PHE. Scale bar $=200 \mu \mathrm{V}, 10$ seconds. $\mathbf{F}$.

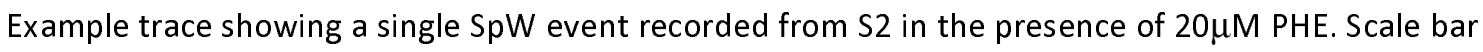
$=100 \mu \mathrm{V}, 200$ milliseconds. ${ }^{*}$ indicates $\mathrm{P}<0.05 .{ }^{* *}$ indicates $\mathrm{P}<0.001$.

Figure 5. LEV supresses SpW induced by peptidergic disinhibition. A. Bar chart showing the reduction of $\mathrm{SpW}$ number by $20 \mu \mathrm{M}$ LEV, when $\mathrm{SpW}$ is induced by the peptidergic disinhibition 
model. Clear bar shows peptidergic disinhibition alone, blue bar show peptidergic disinhibition in the presence of $20 \mu \mathrm{M}$ LEV. B. Bar chart showing the reduction of SpW amplitude by $20 \mu \mathrm{M}$ LEV, when $\mathrm{SpW}$ is induced by the peptidergic disinhibition model. Clear bar shows peptidergic disinhibition alone, blue bar show peptidergic disinhibition in the presence of $20 \mu \mathrm{M}$ LEV. C. Example trace showing SpW activity recorded from S2 following peptidergic disinhibition. D. Example trace showing a SpW activity recorded from S2 following peptidergic disinhibition in the presence of $20 \mu \mathrm{M}$ LEV. Scale bar $=100 \mu \mathrm{V}, 10$ seconds. PHE. Scale bar $=100 \mu \mathrm{V}, 200$ milliseconds. ${ }^{*}$ indicates $\mathrm{P}<0.05 .{ }^{* *}$ indicates $\mathrm{P}<0.001$. 

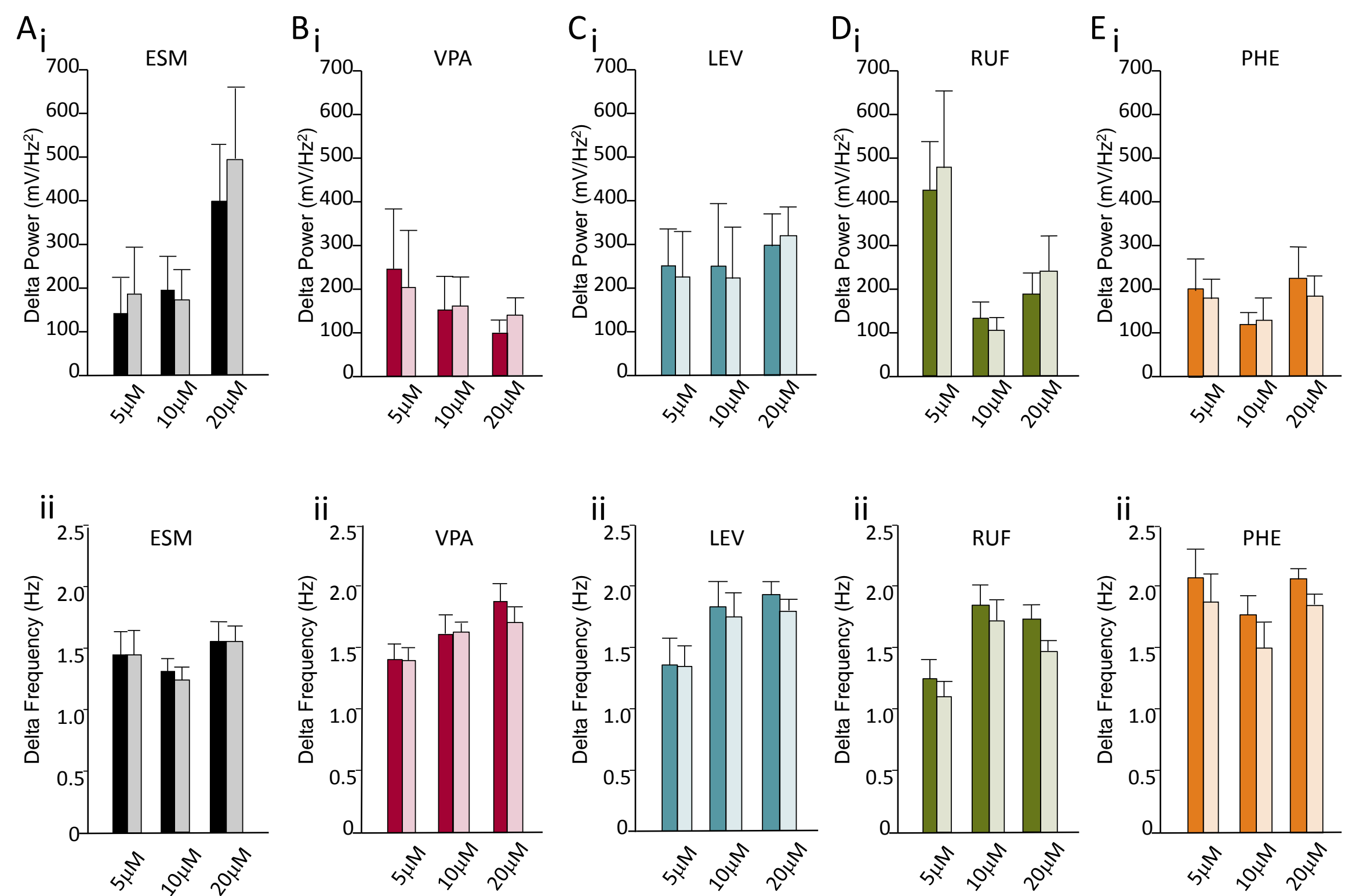
A
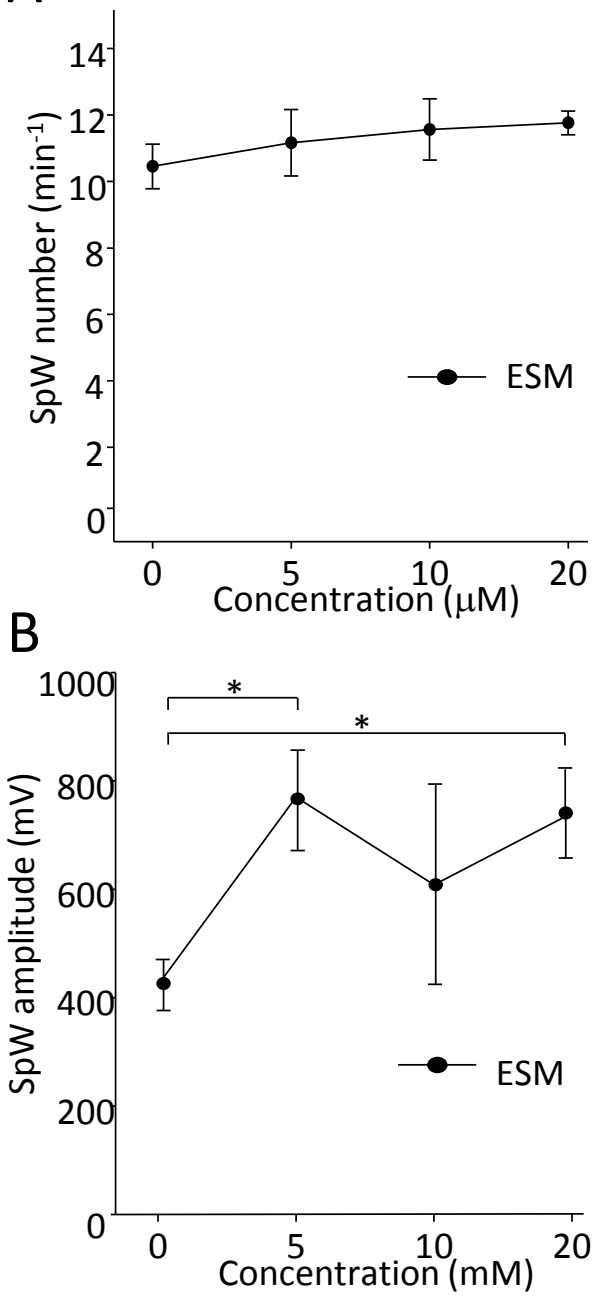

E
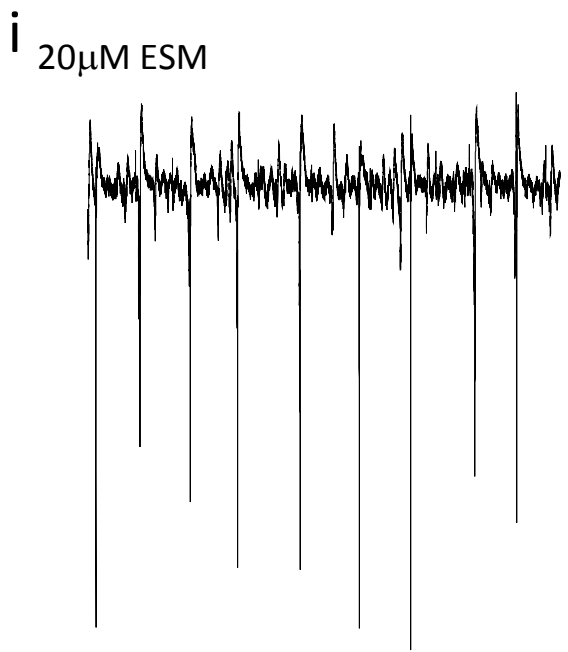

C

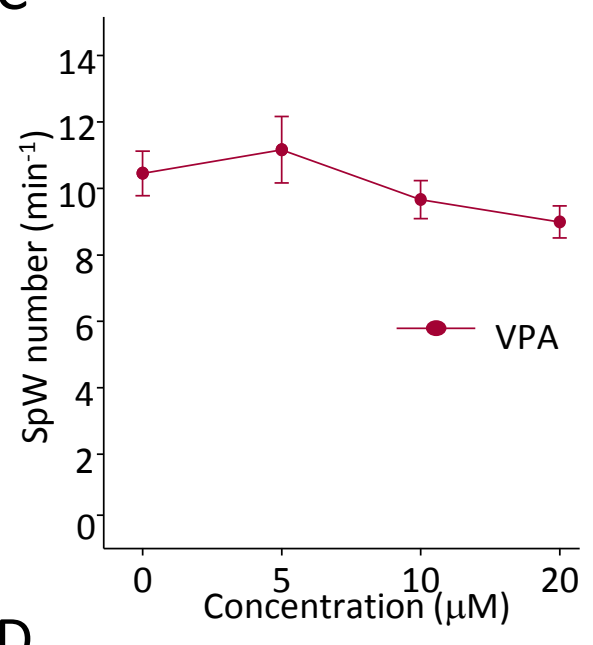

D

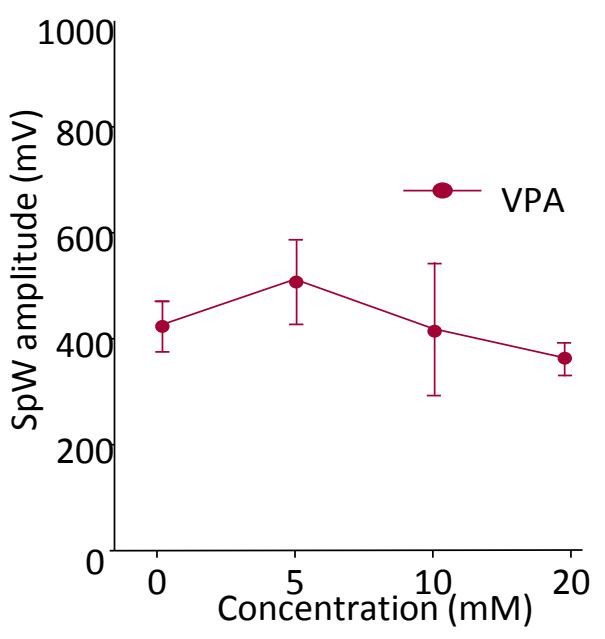

ii $20 \mu \mathrm{M}$ VPA

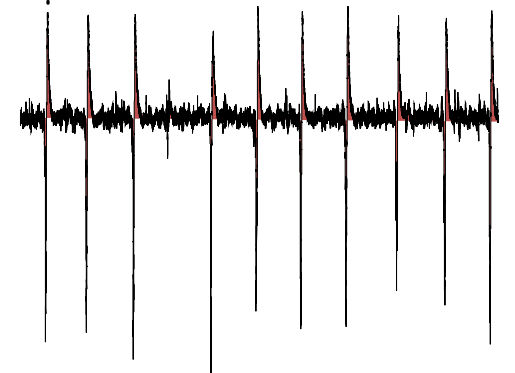


A

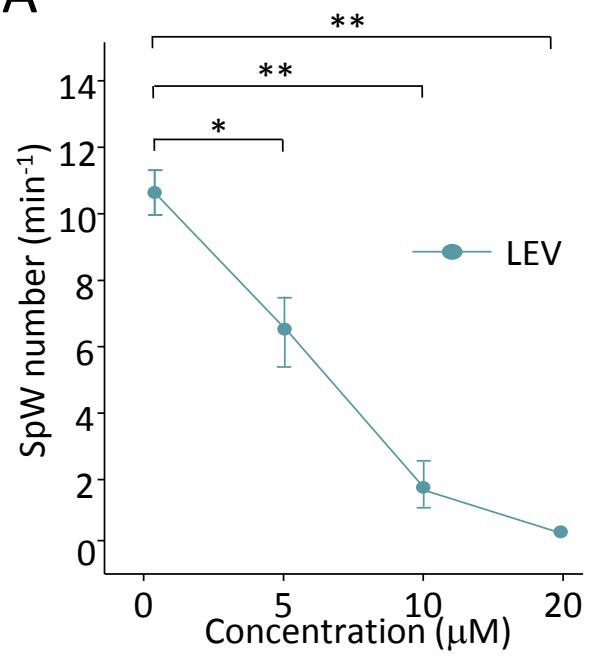

B

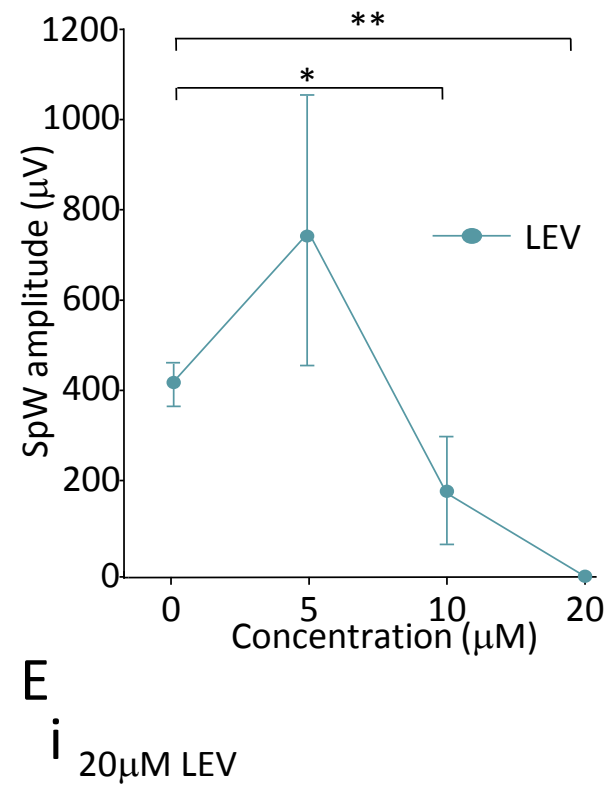

C

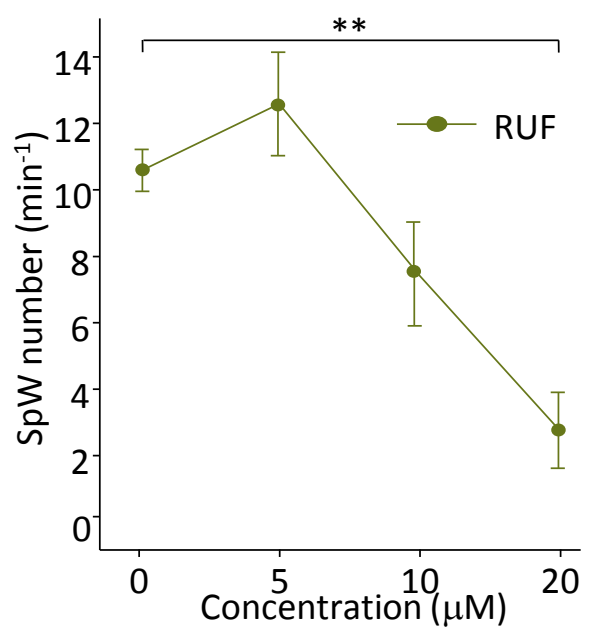

D

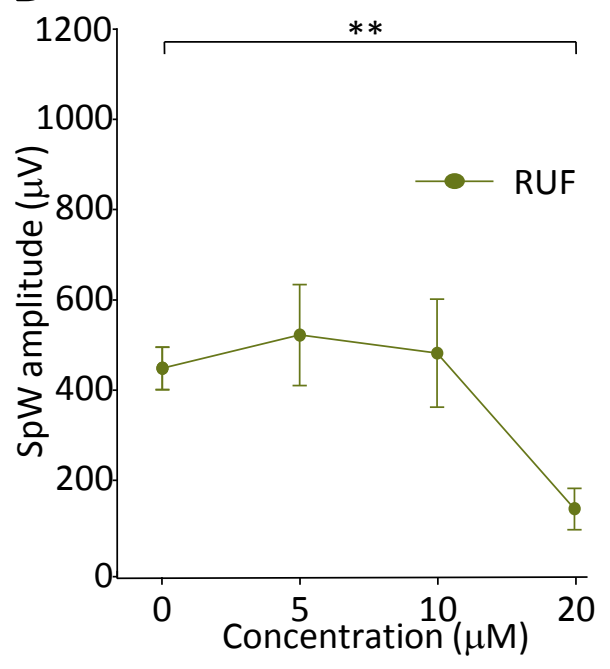

ii $20 \mu \mathrm{M}$ RUF 
A

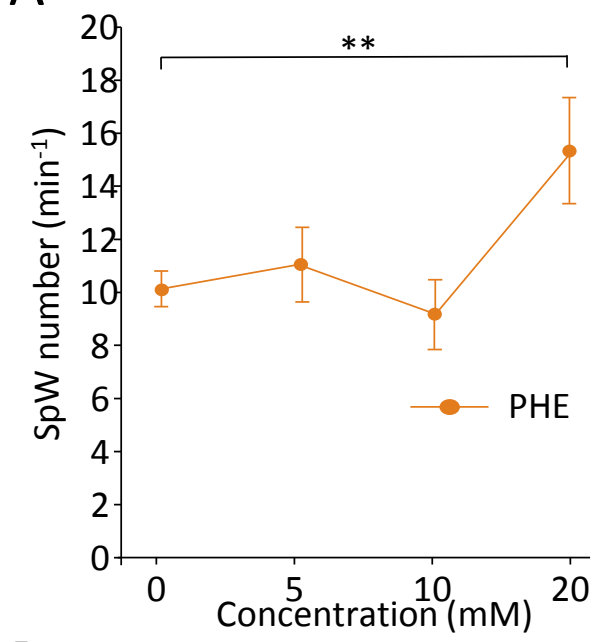

B

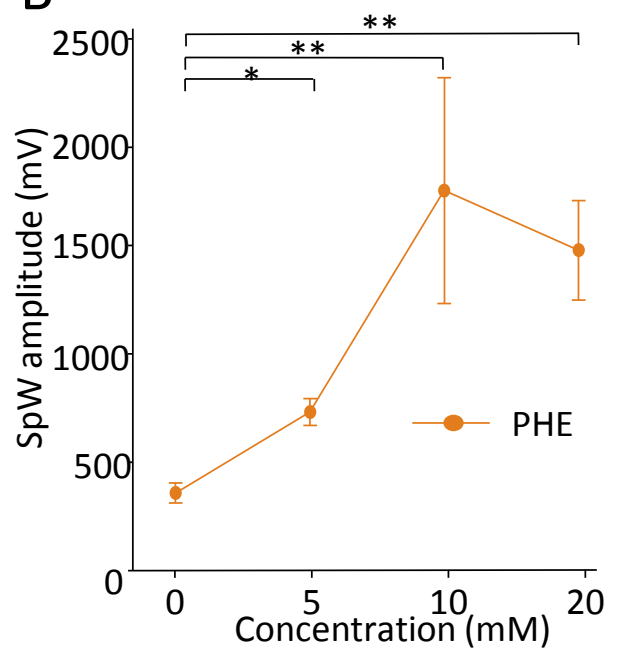

C

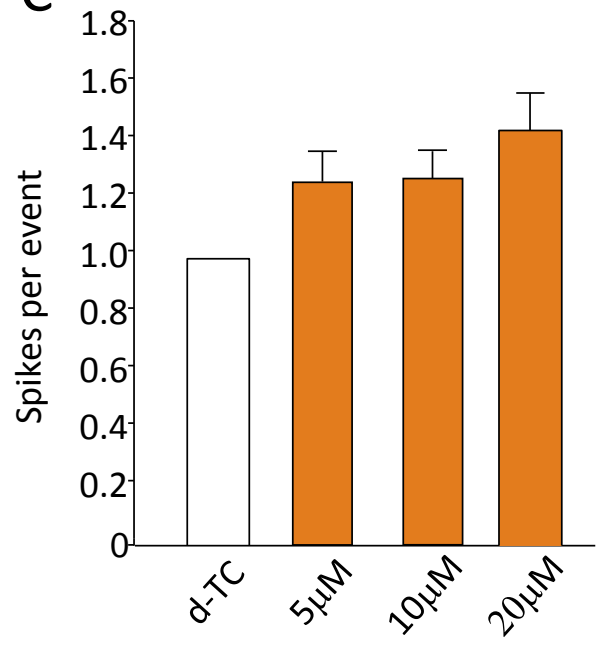

D

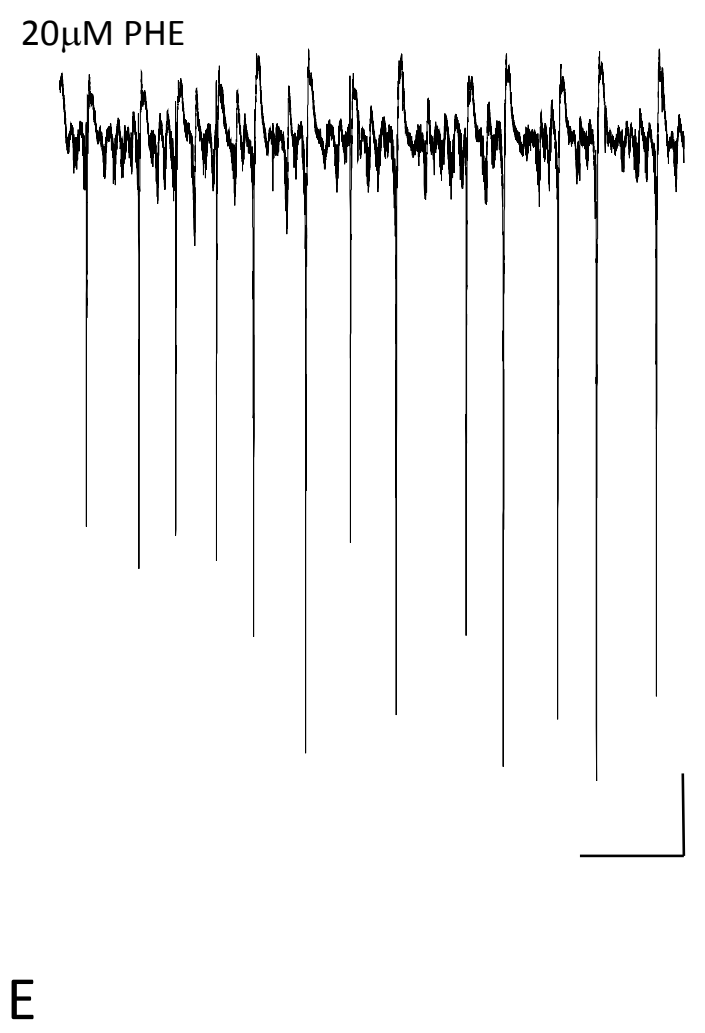

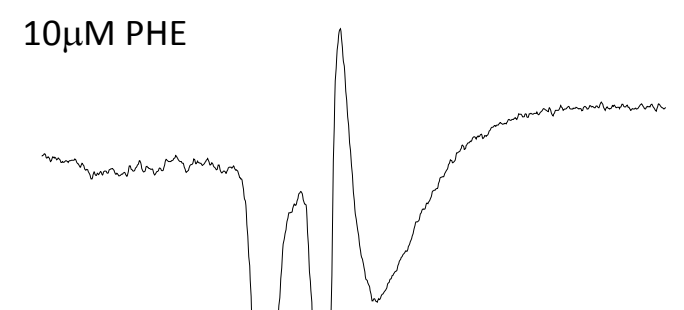


A

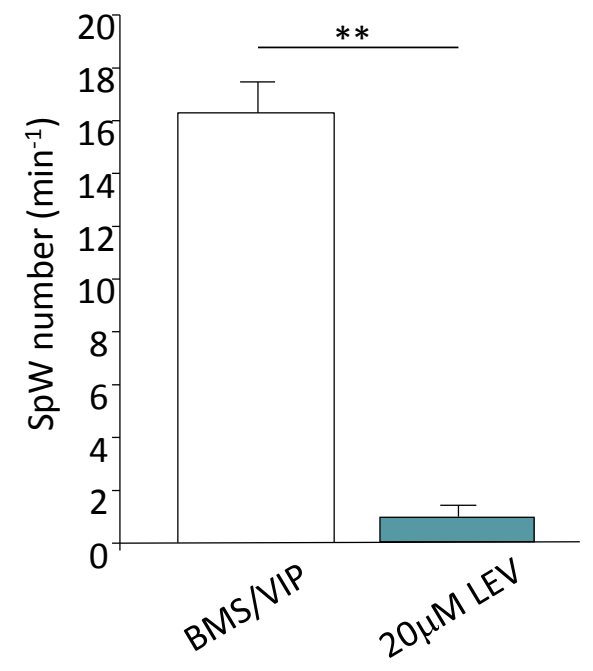

B

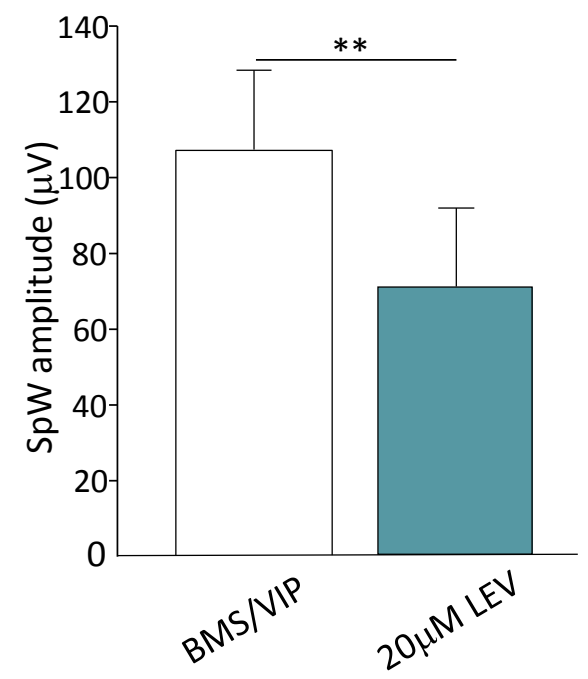

C

$10 \mu \mathrm{M}$ BMS $193885+1 \mu \mathrm{M}$ VIP

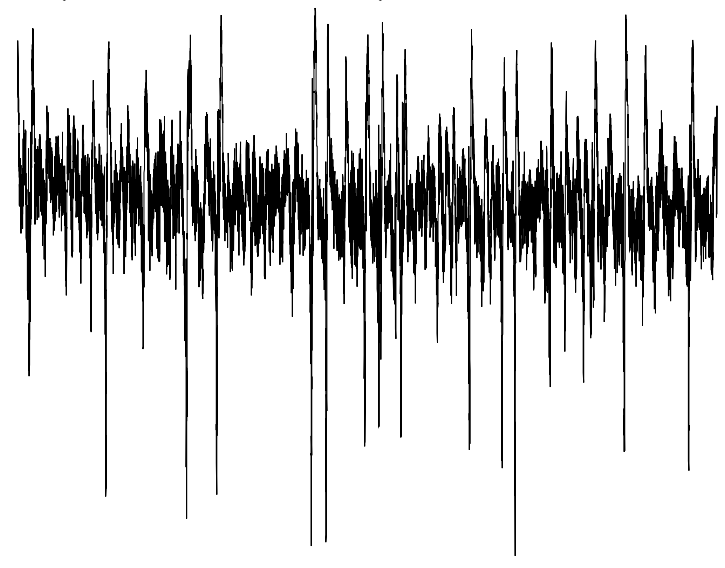

D

$20 \mu \mathrm{M}$ LEV

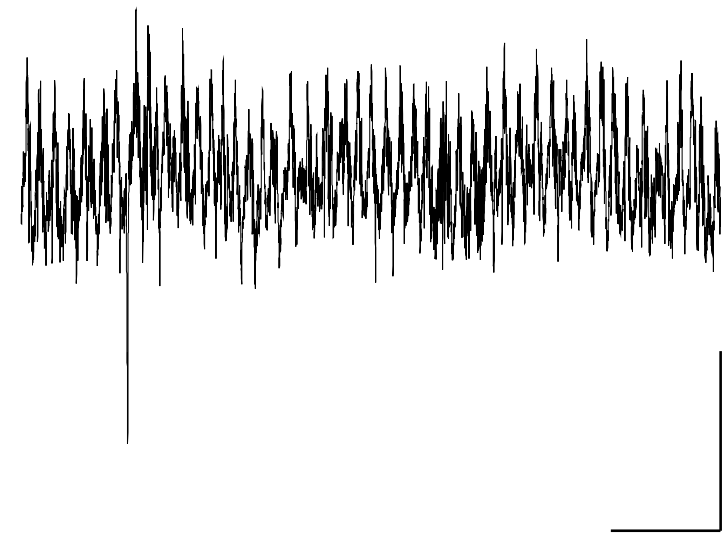

